$t=t_{1} s_{1}$. Since $\left(t_{1} s_{1}\right)^{n_{1}}=s_{1}{ }_{1}$ is in $(s)$, and $n_{1}$ is prime to the order of $s_{1}, s_{1}$ is a power of $s$. Thus $t_{1}$, as well as $t$, corresponds to $t^{\prime}$ in the isomorphism of $G$ with $G /(s)$; but $t_{1}$ and $t^{\prime}$ are of the same order $n_{1}$. Every element of $G /(s)$ whose order is a divisor of $m / m_{1}$ corresponds to an element of $G$ whose order is a divisor of $m$. It follows that $t^{\prime}$, and hence every element of $G /(s)$ whose order divides $n$, is commutative with every element whose order divides $m / m_{1}$. Hence $G /(s)$, being of order $<m n$, contains an invariant subgroup of order $n$. The corresponding subgroup of $G$, being of order $m_{1} n<m n$, also contains an invariant subgroup of order $n$.

Thus in all cases $G$ contains a subgroup of order $n$. Similarly $G$ contains a subgroup of order $m$. $G$ is evidently the direct product of these two subgroups.

HaRVARD UNIVERSTTY

\title{
A CUBIC CURVE CONNECTED WITH TWO TRIANGLES
}

\section{BY H. BATEMAN}

1. Introduction. If $A B C, X Y Z$ are two triangles, a cubic curve $\Gamma_{3}$ may be associated with them as follows.* Let $(P Q, R S)$ denote the point of intersection of the lines $P Q, R S$; then $\Gamma_{3}$ is the locus of a point $O$ such that $(O A, Y Z),(O B, Z X)$, $(O C, X Y)$ are collinear and also the locus of a point $O$ for which $(O X, B C),(O Y, C A),(O Z, A B)$ are collinear. In fact when one set of three points is collinear the other set of three is also collinear. Take $A B C$ as triangle of reference and let the points $X, Y, Z$ have coordinates $\left(x_{1}, x_{2}, x_{3}\right),\left(y_{1}, y_{2}, y_{3}\right)$, $\left(z_{1}, z_{2}, z_{3}\right)$ respectively, then if $(\alpha, \beta, \gamma)$ are current coordinates

* H. Grassmann, Die lineale Ausdehnungslehre, 1844, p. 226. The corresponding quartic surface connected with two tetrahedra is mentioned by H. Fritz, Pr. Ludw. Gymn. Darmstadt [reference taken from Jahrbuch der Fortschritte der Mathematik, vol. 21 (1889), p. 725] and by C. M. Jessop, Quartic Surfaces, Cambridge, 1916, p. 189. 
the condition for the collinearity of the second sets of three points may be expressed in the form

$$
\begin{aligned}
\left(\gamma x_{1}-\alpha x_{3}\right) & \left(\alpha y_{2}-\beta y_{1}\right)\left(\beta z_{3}-\gamma z_{2}\right) \\
& =\left(\alpha x_{2}-\beta x_{1}\right)\left(\beta y_{3}-\gamma y_{2}\right)\left(\gamma z_{1}-\alpha z_{3}\right) .
\end{aligned}
$$

This equation represents a cubic curve passing through the points* $A, B, C, X, Y, Z,(B C, Y Z),(C A, Z X),(A B, X Y)$, ( $C Y, B Z),(A Z, C X),(B X, A Y)$. There is evidently only one cubic curve passing through all these points, and since a cubic through all these points is obtained for the locus when the three points $(O A, Y Z),(O B, Z X),(O C, X Y)$ are collinear, $\Gamma_{3}$ must be the locus in both cases.

This is easily verified analytically. If $X_{1}, X_{2}$, etc. denote the co-factors of the constituents $x_{1}, x_{2}$, etc. in the determinant

$$
\Delta=\left|\begin{array}{lll}
x_{1} & x_{2} & x_{3} \\
y_{1} & y_{2} & y_{3} \\
z_{1} & z_{2} & z_{3}
\end{array}\right|,
$$

the equation of the locus in the first case may be expressed in the form

$$
\left|\begin{array}{ccc}
\beta X_{2}+\gamma X_{3} & -\beta X_{1} & -\gamma X_{1} \\
-\alpha Y_{2} & \alpha Y_{1}+\gamma Y_{3} & -\gamma Y_{2} \\
-\alpha Z_{3} & -\beta Z_{3} & \alpha Z_{1}+\beta Z_{2}
\end{array}\right|=0
$$

and the equivalence of the two equations is easily verified with the aid of a number of identities, one of which is

$$
\begin{aligned}
2 X_{1} Y_{2} Z_{3}- & Y_{1} X_{3} Z_{2}-Z_{1} X_{2} Y_{3} \\
& =\Delta\left(2 x_{1} y_{2} z_{3}-y_{1} x_{3} z_{2}-z_{1} x_{2} y_{3}\right) \\
& =\Delta\left(x_{1} X_{1}+y_{2} Y_{2}+z_{3} Z_{3}-\Delta\right) .
\end{aligned}
$$

2. Salmon's Projective Invariant. By a well known theorem due to Salmon the cross ratio of the four tangents to a cubic from the point on the curve is constant and represents

* The properties of this configuration of 12 points and some degenerate cases of the cubic curve are discussed by H. M. Taylor, Proceedings of the London Society, (1), vol. 28 (1897), pp. 545-555. 
an invariant of the curve. In the present case this invariant will be an invariant of the two triangles* and it seems worth while to calculate it. A direct calculation is laborious when use is made of the equation of the curve in one of the forms already given; the work may be simplified, however, by noticing that the four lines $B C, Y Z, B Z, C Y$ form a complete quadrilateral whose six vertices lie on the curve. Denoting the equations of these lines by $\alpha=0, \theta=0, \phi=0, \psi=0$ respectively, the equation of the curve may be written in the form

$$
\frac{A}{\alpha}+\frac{B}{\theta}+\frac{C}{\phi}+\frac{D}{\psi}=0 \text {, }
$$

where the identical relation between $\alpha, \theta, \phi, \psi$ is

and

$$
\alpha+\theta+\phi+\psi=0,
$$

$$
\begin{array}{ll}
A=y_{2} z_{1}^{2} X_{2}^{2} Z_{3}, & B=y_{1}^{2} z_{3} X_{3}^{2} Y_{2}, \\
C=y_{1}^{2} z_{1}^{2} X_{1} \Delta, & D=x_{1} X_{2}^{2} X_{3}^{2} .
\end{array}
$$

If $\lambda$ is one of the cross ratios of the four tangents, $\lambda$ is connected with the coefficients $A, B, C, D$ by the relation $\dagger$

$$
(\lambda+1)^{2}(\lambda-2)^{2}\left(\lambda-\frac{1}{2}\right)^{2} I^{3}=27\left(\lambda^{2}-\lambda+1\right)^{3} J^{2},
$$

where $I$ and $J$, the usual invariants of a biquadratic equation, are given by the equations

$$
\begin{gathered}
12 I=\Theta^{2}-48 A B C D, \quad 216 J=\Theta\left[72 A B C D-\Theta^{2}\right], \\
I^{3}-27 J^{2}=A^{2} B^{2} C^{2} D^{2}\left[\Theta^{2}-64 A B C D\right],
\end{gathered}
$$

where

$$
\begin{aligned}
\Theta^{2}=A^{2}+B^{2}+C^{2}+ & D^{2}-2 B C-2 C A-2 A B \\
& -2 A D-2 B D-2 C D .
\end{aligned}
$$

* A complete set of invariants of two triangles is given in a paper by D. D. Leib, Transactions of this Society, vol. 10 (1909), p. 361. In our case there is a correspondence between the vertices of the triangles and the invariant theory is a little different.

$\dagger$ The coefficient $m$ in the canonical equation $x^{3}+y^{3}+z^{3}+6 m x y z=0$ is connected with $I$ and $J$ by the relation

$$
\frac{64 m^{3}\left(m^{3}-1\right)^{3}}{I^{3}}=\frac{\left(8 m^{6}+20 m^{3}-1\right)^{2}}{27 J^{2}}
$$

See H. Hilton, Plane Algebraic Curves, Oxford, 1920, p. 235. 
Substituting the expressions for $A, B, C, D$, we find that

$$
\begin{gathered}
\Theta=y_{1}^{2} z_{1}^{2} X_{2}^{2} X_{3}^{2}\left[\left(x_{3} y_{1} z_{2}-x_{2} y_{3} z_{1}\right)^{2}-4 \Delta x_{1} y_{2} z_{3}-4 X_{1} Y_{2} Z_{3}\right], \\
A B C D=y_{1}^{4} z_{1}^{4} X_{2}^{4} X_{3}^{4}\left[x_{1} X_{1} y_{2} Y_{2} z_{3} Z_{3} \Delta\right] .
\end{gathered}
$$

The nature of the cubic is thus seen to depend on the value of the single invariant

$$
K=\frac{\left[\left(x_{3} y_{1} z_{2}-x_{2} y_{3} z_{1}\right)^{2}-4 \Delta x_{1} y_{2} z_{3}-4 X_{1} Y_{2} Z_{3}\right]^{2}}{x_{1} y_{2} z_{3} X_{1} Y_{2} Z_{3} \Delta} .
$$

The cubic has a double point when this invariant is either infinite or 64. When $\Theta=0$ we have $J=0$ and the four tangents form a harmonic pencil. If $A, B, C, Y$, and $Z$ are given, the locus of $X$ when $\Theta=0$ is a conic. This, however, is not the complete locus when the tangents form a harmonic pencil because $J$ vanishes also when $\Theta^{2}=72 A B C D$ or $K=72$. It should be noticed that the expression $K$ remains the same when we replace each constituent in the determinant by its co-factor. We have in fact an identity of type

$$
\begin{aligned}
X_{3} Y_{1} Z_{2}-X_{2} Y_{3} Z_{1} & =\Delta\left(z_{3} Z_{3}-y_{2} Y_{2}+x_{3} X_{3}-x_{2} X_{2}\right) \\
& =\Delta\left(x_{3} y_{1} z_{2}-x_{2} y_{3} z_{1}\right),
\end{aligned}
$$

with the aid of which the preceding remark is easily verified.

Quantities that possess this property may be called invariants of the determinant. To find such quantities we may commence by finding pseudo-invariants, that is, quantities which retain the same form except for a power of the determinant. The quantities $x_{1} X_{1}, x_{2} X_{2}, x_{3} X_{3}, y_{1} Y_{1}, y_{2} Y_{2}, y_{3} Y_{3}$, $z_{1} Z_{1}, z_{2} Z_{2}, z_{3} Z_{3}$ all possess this last property when considered as functions of the constituents $x_{1}, x_{2}$, etc. and a similar remark may be made in the case of a determinant of higher order. For the general case of a determinant of order $n \mathrm{I}$ do not know definitely how many pseudo-invariants of degree $n$ in the constituents are linearly independent. A consideration of the cases $n=2,3$, and 4 leads to the conclusion that this number $N$ may be given by the formula

$$
N=(n-1)^{2}+1 .
$$


When $n=2$, it is evident that $N=2$. When $n=3$ it appears that $N=5$, for each of the nine quantities $x_{1} X_{1}, x_{2} X_{2}, x_{3} X_{3}, y_{1} Y_{1}$, $y_{2} Y_{2}, y_{3} Y_{3}, z_{1} Z_{1}, z_{2} Z_{2}, z_{3} Z_{3}$ can be expressed as a linear function of the five linearly independent quantities $x_{1} X_{1}, x_{2} X_{2}, y_{1} Y_{1}$, $y_{2} Y_{2}, \Delta$.

In the case of a determinant of the fourth order

$$
\left|\begin{array}{llll}
x_{1} & x_{2} & x_{3} & x_{4} \\
y_{1} & y_{2} & y_{3} & y_{4} \\
z_{1} & z_{2} & z_{3} & z_{4} \\
t_{1} & t_{2} & t_{3} & t_{4}
\end{array}\right|
$$

the problem is more complex because in addition to the pseudoinvariants $x_{s} X_{s}$ etc. there are pseudo-invariants of type $\left(y_{2} z_{3}-y_{3} z_{2}\right)\left(x_{1} t_{4}-x_{4} t_{1}\right)$.

Adopting the notation

$$
\left(\begin{array}{l}
y z \\
14
\end{array}\right)=\left(y_{2} z_{3}-y_{3} z_{2}\right)\left(x_{1} t_{4}-x_{4} t_{1}\right)=\left(\begin{array}{l}
x t \\
23
\end{array}\right),
$$

we observe that the relations of type

$$
\begin{aligned}
& y_{1} Y_{1}=x_{2} X_{2}+\left(\begin{array}{l}
x t \\
12
\end{array}\right)+\left(\begin{array}{l}
z x \\
12
\end{array}\right)-\left(\begin{array}{l}
x y \\
31
\end{array}\right)-\left(\begin{array}{l}
x y \\
14
\end{array}\right) \\
& y_{2} Y_{2}=x_{1} X_{1}+\left(\begin{array}{l}
z x \\
12
\end{array}\right)+\left(\begin{array}{l}
x t \\
12
\end{array}\right)+\left(\begin{array}{l}
x y \\
24
\end{array}\right)+\left(\begin{array}{l}
x y \\
23
\end{array}\right), \\
& y_{3} Y_{3}=x_{4} X_{4}+\left(\begin{array}{l}
x t \\
34
\end{array}\right)+\left(\begin{array}{l}
z x \\
34
\end{array}\right)-\left(\begin{array}{l}
x y \\
31
\end{array}\right)-\left(\begin{array}{l}
x y \\
23
\end{array}\right), \\
& y_{4} Y_{4}=x_{3} X_{3}+\left(\begin{array}{l}
x t \\
34
\end{array}\right)+\left(\begin{array}{l}
z x \\
34
\end{array}\right)-\left(\begin{array}{l}
x y \\
14
\end{array}\right)-\left(\begin{array}{l}
x y \\
24
\end{array}\right)
\end{aligned}
$$

indicate that all the pseudo-invariants of type $y_{s} Y_{s}, z_{s} Z_{s}, t_{s} T_{s}$ can be expressed in terms of the pseudo-invariants $x_{1} X_{1}, x_{2} X_{2}$, $x_{3} X_{3}, x_{4} X_{4}$ and the 18 quantities of type

$$
\left(\begin{array}{c}
y z \\
14
\end{array}\right) \text {. }
$$


It would be wrong, however, to suppose that there are 22 linearly independent pseudo-invariants of degree four, for there are the six identities

$$
\begin{aligned}
& x_{1} X_{1}+x_{4} X_{4}=\left(\begin{array}{l}
x y \\
23
\end{array}\right)+\left(\begin{array}{l}
z x \\
23
\end{array}\right)+\left(\begin{array}{l}
x t \\
23
\end{array}\right), \\
& x_{2} X_{2}+x_{4} X_{4}=\left(\begin{array}{l}
x y \\
31
\end{array}\right)+\left(\begin{array}{l}
z x \\
31
\end{array}\right)+\left(\begin{array}{l}
x t \\
31
\end{array}\right), \\
& x_{3} X_{3}+x_{4} X_{4}=\left(\begin{array}{l}
x y \\
12
\end{array}\right)+\left(\begin{array}{l}
z x \\
12
\end{array}\right)+\left(\begin{array}{l}
x t \\
12
\end{array}\right), \\
& x_{2} X_{2}+x_{3} X_{3}=\left(\begin{array}{l}
x y \\
14
\end{array}\right)+\left(\begin{array}{l}
z x \\
14
\end{array}\right)+\left(\begin{array}{l}
x t \\
14
\end{array}\right), \\
& x_{3} X_{3}+x_{1} X_{1}=\left(\begin{array}{l}
x y \\
24
\end{array}\right)+\left(\begin{array}{l}
z x \\
24
\end{array}\right)+\left(\begin{array}{l}
x t \\
24
\end{array}\right), \\
& x_{1} X_{1}+x_{2} X_{2}=\left(\begin{array}{l}
x y \\
34
\end{array}\right)+\left(\begin{array}{l}
z x \\
34
\end{array}\right)+\left(\begin{array}{l}
x t \\
34
\end{array}\right),
\end{aligned}
$$

three relations of type

$$
\Delta=\left(\begin{array}{l}
x y \\
34
\end{array}\right)+\left(\begin{array}{l}
x y \\
14
\end{array}\right)+\left(\begin{array}{l}
x y \\
24
\end{array}\right)+\left(\begin{array}{l}
x y \\
23
\end{array}\right)+\left(\begin{array}{l}
x y \\
31
\end{array}\right)+\left(\begin{array}{l}
x y \\
12
\end{array}\right),
$$

three relations of type

$$
\Delta=\left(\begin{array}{l}
y z \\
12
\end{array}\right)+\left(\begin{array}{l}
z x \\
12
\end{array}\right)+\left(\begin{array}{l}
x y \\
12
\end{array}\right)+\left(\begin{array}{l}
x t \\
12
\end{array}\right)+\left(\begin{array}{l}
y t \\
12
\end{array}\right)+\left(\begin{array}{l}
z t \\
12
\end{array}\right),
$$

and the relation $x_{1} X_{1}+x_{2} X_{2}+x_{3} X_{3}+x_{4} X_{4}=\Delta$. The number of linearly independent pseudo-invariants thus seems to be ten.

California Institute of Technology 